\title{
El Derecho Probatorio y su Torre de Babel. Sobre citas en revistas indexadas*
}

\author{
Rodrigo Coloma Correa**
}

\begin{abstract}
RESUMEN
Este texto analiza las prácticas en la producción de saberes probatorios en contextos jurídico-académicos. Un aspecto a dilucidar es si las teorías y explicaciones que se proponen son o no incrementales. Aquello impacta no solo en la profundidad y en el nivel de detalles de los que se bacen cargo, sino también en el grado de consolidación de la respectiva disciplina (Derecho Probatorio). El estudio de las citas en artículos publicados en revistas chilenas indexadas en WoS (ex ISI) y SCIELO, indica que bay un nivel medio-bajo de cohesión entre sus autores, es decir, la dinámica de producción del conocimiento es parcialmente incremental. Esto se explica, en parte importante, por la dispersión en las perspectivas de análisis (dogmática o teoría del derecho). Para el progreso de la disciplina es importante concordar propósitos y alcanzar consensos conceptuales que propendan la generación de conocimientos lo suficientemente complejos como para satisfacer requerimientos sociales en asuntos de prueba.
\end{abstract}

Citas académicas -Derecho Probatorio - disciplinas jurídicas

\section{Evidence Law and its Tower of Babel}

\begin{abstract}
The paper reviews practices in the creation of legal evidence knowledge under legal-academic contexts. One of the issues studied is whether theories and explanations proposed in those contexts are incremental or not. This aspect impacts not only in the depth and details achieved, but also in the degree of consolidation of the very same legal discipline (Evidence Law). The study of citations in papers published in Chilean Journals indexed in WoS (former ISI) and SCIELO shows that there is a mid-low level of cohesion among scholars. Therefore, the dynamic in the production of knowledge is partially incremental. The dispersion in analysis perspectives (legal theory or jurisprudence) explains, for the most part, the aforementioned finding. In order for the legal discipline to progress, it is important to agree among aims and to reach conceptual consensus so as to favor the creation of knowledge complex enough to satisfy social requirements in evidence issues.
\end{abstract}

Academic citation - evidence law - legal disciplines

* Este trabajo presenta resultados del proyecto financiado por el Fondo Nacional de Desarrollo Científico y Tecnológico, FONDECYT 1140986 "Conceptos fundamentales del Derecho Probatorio en Chile". El presente artículo se relaciona estrechamente con otros artículos. Así, le antecede "Las disciplinas jurídicas y su reinvención" y están en una fase de preparación otros textos que refieren a: i) las particularidades de la enseñanza del Derecho Probatorio; ii) bases de un modelo conceptual para decidir hechos; y iii) conceptos y formas de razonamiento para decidir hechos; y iv) los usos de los estándares de prueba.

** Doctor en Derecho, Universidad Carlos III de Madrid. Profesor de la Facultad de Derecho de la Universidad Alberto Hurtado. Correo electrónico: rcoloma@uahurtado.cl.

Artículo recibido el 28.12.2015 y aceptado para su publicación el 30 de agosto de 2016. 
Pero el Señor bajó a ver la ciudad y la torre que los hombres estaban construyendo, y dijo: "Si esta es la primera obra que realizan, nada de lo que se propongan hacer les resultará imposible, mientras formen un solo pueblo y todos hablen la misma lengua. Bajemos entonces, y una vez alli, confundamos su lengua, para que ya no se entiendan unos a otros".

Génesis $11,5-7$

\section{EL PROBLEMA I}

\section{Sobre el escenario que enfrenta el Derecho Probatorio}

V arios cambios en las últimas décadas desaconsejan no tomarse suficientemente en serio los problemas que aquejan a la producción y a la validación de los saberes probatorios. Algunos de estos cambios han sido normativos y conciernen tanto a una reconfiguración de los sistemas de valoración de la prueba como también a una progresiva apertura hacia la admisibilidad de una mayor gama de piezas de información ${ }^{2}$. Otros tienen que ver con la disponibilidad de mejores tecnologías para evitar errores, como es el caso de la prueba de ADN y de algunas otras pruebas periciales ${ }^{3}$. Se favorece, así, una reconfiguración de los saberes probatorios, mucho más abiertos a incorporar aportes de disciplinas no jurídicas y, a la vez, atentos a lo que ocurre en

${ }^{1}$ Este texto no habría sido posible sin la valiosa colaboración de Victoria Martínez Placencia. Ella estuvo a cargo de construir la base de datos de artículos académicos, desde la que se extrae la información requerida para completar tablas que se insertan en este trabajo. Una versión preliminar de este texto fue discutida en el Seminario 3 de la Universidad Alberto Hurtado: agradezco las valiosas observaciones que me fueron hechas por Jean Pierre Matus, Claudio Agüero, Mauricio Duce, Claudio Fuentes y los restantes asistentes. Agradezco, además, la minuciosa lectura de Daniela Accatino y sus agudos comentarios. Los errores que persisten son, por cierto, de mi responsabilidad.

${ }^{2}$ Como se sabe, la sana crítica está sustituyendo modelos de valoración de la prueba que antaño imperaban. A ello se suma una decidida política en favor de que toda prueba debe en principio ser admitida, a menos que sea expresamente excluida. La tecnología tiene las puertas abiertas para penetrar con fuerza en los salas de audiencia de los tribunales de justicia. Los cambios normativos han suscitado bastante interés en la comunidad de jueces y abogados estimulándose, de esa manera, un incremento de textos explicativos, como también de actividades de capacitación. Respecto de esto último, es necesario notar además el impacto del tránsito desde la escrituración hacia la oralidad. Esta obliga a jueces y abogados a reaccionar de inmediato, ello implica que quedarán expuestos a la crítica si no dominan las técnicas y saberes requeridos para superar correctamente cualquier situación inesperada. Cfr. Lempert, R., “The New Evidence Scholarship: Analyzing the Process of Proof”, en 66 Boston University Law Review, 1986, pp. 439-440.

${ }^{3}$ Así, en un texto reciente se afirma que: "En el último tiempo se ha venido instalando una creciente preocupación en nuestro país frente a la posibilidad que el sistema de justicia penal pueda cometer errores y, más específicamente, condenar a personas inocentes. Esta preocupación es consistente con lo que ha ocurrido en el ámbito comparado en las últimas décadas, tanto en el mundo anglosajón como europeo continental, en el que el tema se ha transformado en una enorme fuente de debate...". Duce, M., "Algunas lecciones a partir de cuatro casos de condena de inocentes en Chile", en Revista de Derecho de la Universidad Católica del Norte, Año 22, No 1, 2015, pp. 150-151. 
otras tradiciones jurídicas ${ }^{4}$. Una tercera familia de cambios -menos evidente que las anteriores- se relaciona con la producción de artículos académicos, estos vienen experimentando un sostenido crecimiento en las últimas décadas. El cambio cuantitativo, en ocasiones, ha ido de la mano de uno de carácter cualitativo. Aun cuando no en forma contundente, se advierte la depuración de un género académico que progresivamente satisface nuevos requerimientos metodológicos 5 . Hay una estructura textual que se va poco a poco homogeneizando, a la vez que se van ampliando las fuentes de información disponibles (aunque no necesariamente su uso) ${ }^{6}$.

Las alteraciones en el escenario de lo probatorio propician un acercamiento entre quienes tradicionalmente se han ocupado de asuntos dogmático-políticos y quienes se han interesado por asuntos epistémico-decisionales. De esta manera, sujetos provenientes de disciplinas muy distintas como lo son el Derecho Procesal y la Teoría del Derecho (entre otras) entienden que coinciden parcialmente en su campo de trabajo-investigación (en adelante, el "campo") ${ }^{7}$, aun cuando sus métodos de trabajo sean diferentes. El espacio común en que aquí se llama la atención es el del Derecho Probatorio ${ }^{8}$.

La locución Derecho Probatorio no es regularmente utilizada entre jueces y abogados y, por tanto, requiere de algunas precisiones ${ }^{9}$. El Derecho Probatorio, en lo que sigue, será entendido como una (proto) disciplina que se hace cargo de un campo que incluye: i) conjeturas sometidas a contrastación en procesos adjudicativos ("hechos a probar"); ii) prácticas de producción de pruebas en contextos jurídicos ("rendición de pruebas"); iii) modelos que pueden/deben utilizarse para evaluar las pruebas rendidas en distintos casos ("valoración de la prueba"); y iv) hechos que institucionalmente son

${ }^{4}$ Desde hace algún tiempo se observa un creciente interés por lo que puedan decir epistemólogos, psicólogos, etcétera. Hay, asimismo, una creciente atención en lo que sucede especialmente en la cultura del Common Law, reconociéndose los mayores avances que ha tenido respecto de la nuestra.

${ }^{5}$ Este fenómeno en parte se ha visto influido por la disponibilidad de fondos públicos para la investigación, por la vía de postulación y adjudicación de proyectos Fondecyt. Los investigadores han tenido que ceñirse a un formato al que no estaban acostumbrados hasta hace algunos años (formulación de hipótesis, determinación de marco teórico y de metodología de trabajo, control de pares, etcétera). Un producto especialmente valorado es la publicación de artículos en revistas indexadas, las que también exigen el cumplimiento de reglas en la producción científica.

${ }^{6}$ Por cierto, esto no garantiza que los nuevos textos sean más interesantes o más útiles que los de antaño.

${ }^{7}$ Con "campo de trabajo-investigación" refiero a un espacio de influencia compartido por jueces, abogados y académicos. El campo no se reduce al objeto de estudio de un grupo de sujetos, sino que asume que hay luchas por alcanzar una posición hegemónica y convertir, expulsar o ignorar a quienes no se sometan a sus exigencias. La noción de campo es clave en la obra de Pierre Bourdieu. Bourdieu, P., "Elementos para una sociología del campo jurídico", en Bourdieu, P., Teubner, G.. La fuerza del derecho, Universidad de los Andes y otras, Bogotá, 2000 , en especial p. 155, nota del traductor.

${ }^{8}$ Para la situación en países del Common Law ver Twining, W., "Moving Beyond Law: Interdisciplinarity and the Study of Evidence", en Dawid, P., Twining, W., Vasilaki, M. (eds.), Evidence Inference and Enquiry, The British Academy-Oxford University Press, 2011, pp. 73-118.

9 Por cierto, tampoco es desconocida entre nosotros. El año 1965 Niceto Alcalá-Zamora publicó por la Universidad de Concepción una colección de trabajos sobre distintos asuntos de prueba a la cual denominó "Estudios de Derecho Probatorio". Alcalá-Zamora, N., Estudios de Derecho Probatorio, Universidad de Concepción, Concepción, 1965. 
posibles de considerar como probados o no probados ("decisión y justificación de los hechos probados").

Desde la perspectiva de los especialistas del Derecho Procesal una redefinición como la recién sugerida podría desencadenar algún grado no menor de resistencia; ello en cuanto les impulsa a hacerse cargo de algunos asuntos difíciles que, de acuerdo con la visión más tradicional, resulta fácil desentenderse. Esto, por cierto, tiene repercusiones importantes, en cuanto implica alterar ciertas dinámicas de trabajo establecidas. La forma usual de operar -hasta hace poco tiempo- explica, por ejemplo, el éxito de distinciones dudosas como la que, según se enseña, se da entre verdad material y verdad formal. En mi opinión, dicha distinción no refleja un escepticismo generalizado de los procesalistas acerca de las posibilidades de aproximarse a una forma de conocimiento profundo (o interesante) de la realidad ${ }^{10}$, sino simplemente da cuenta de que hay problemas de naturaleza epistémica de los cuales ellos no tienen por qué hacerse cargo ${ }^{11}$. Al diluirse el dualismo entre verdad material y verdad material -como podría ocurrir al reconfigurarse el campo en los términos recién señalados- los procesalistas se verían forzados a adoptar una perspectiva de análisis no puramente dogmática, sino una que, entre otras cosas, se haga cargo directamente de algunas expectativas epistémicas y de problemas de coherencia narrativa que con frecuencia afectan al texto de las sentencias ${ }^{12}$. En pocas palabras, la redefinición del campo obliga a los procesalistas a ampliar el universo de problemas y el foco de su análisis, lo que -creo- redundaría en aportes más interesantes ${ }^{13}$.

Desde la perspectiva de los teóricos del derecho acoger la denominación "Derecho Probatorio" - la que aparece notoriamente comprometida con la generación de teorías y explicaciones de uso directo en los procesos judiciales- se aleja de lo que de ellos tradicionalmente se ha esperado, esto es hacer metajurisprudencia u otras cuestiones

${ }^{10}$ Marina Gascón, en consonancia con otros autores, indica que la separación entre la ciencia procesal y la epistemología, en los términos observables hasta ahora, es un fenómeno que se produce en la época postilustrada. Gascón, M., Los hechos en el Derecho. Bases argumentales de la prueba. Marcial Pons, Madrid, 1999, pp. 33-41.

${ }^{11}$ Tradicionalmente se ha entendido que el campo de investigación de los cultores de distintas disciplinas se encuentra firmemente determinado por disposiciones jurídicas que es menester optimizar. Esta visión, aun cuando incompleta, presta no poca utilidad para la neutralización de los intentos por aplicar normas ideales no explícitas (apelación directa a la justicia). Así, por ejemplo, los penalistas distinguen entre argumentos o propuestas de lege lata y de lege ferenda, para enfatizar que solo mediante las últimas se están escabullendo de las tareas que les son más propias (política jurídica). El caso de los problemas probatorios es distinto, pues el conjunto de disposiciones es parco. Mediante este se intenta establecer cortapisas a ciertas formas de operar que no resultan satisfactorias en los contextos judiciales, no pretendiéndose que las disposiciones normativas lo cubran todo (incluso, en sistemas de prueba tasada). Así las cosas, distinguir entre verdad material y formal con miras a desentenderse de exigencias que resultan posibles de satisfacer, sin apartarse de lo que se dice en las normas jurídicas, no tiene mucho sentido.

${ }^{12}$ Solo, a modo de ejemplo, los estudios de Derecho Probatorio no se hacen cargo de cómo debiesen ser reconstruidas en las sentencias las declaraciones de los testigos y peritos, lo que es relevante a los efectos de su coherencia narrativa.

${ }^{13} \mathrm{Al}$ menos entre algunos procesalistas la preocupación está muy presente y algunas de sus lecturas así lo demuestran. Ellos - como se verá- están leyendo con atención a algunos autores con enfoques muy amplios y cuyas propuestas no son fácilmente transferibles hacia las disposiciones normativas locales. 
por el estilo. Para los teóricos resultaría más cómodo hablar de una Teoría General de la Prueba que, como tal, no se encuentre amarrada a las contingencias de los sistemas jurídicos. Acorde a lo expresado precedentemente -esto es, los cambios de escenarios en los asuntos de prueba-, surgen razones para que las propuestas de los dogmáticos sean optimizadas por los teóricos haciendo uso de las posibilidades que arroja el análisis conceptual o metodológico en contextos normativos concretos.

El escenario actual del Derecho Probatorio resulta especialmente propicio para acoger diferentes perspectivas de análisis. De lo que se trata, entonces, es de impulsar, una práctica de intercambios que favorezca lo complejo de los saberes, en términos de que resulten especialmente útiles para enfrentar exitosamente los problemas propios del campo ${ }^{14}$.

\section{Plan de trabajo}

Lo que a continuación se desarrolla tiene pretensiones eminentemente descriptivas, sin perjuicio de que a nivel de conclusiones sea complementado con sugerencias de cursos de acción. De lo que se trata, es de levantar información acerca de lo que está ocurriendo a nivel de producción de saberes probatorios e, indirectamente, conocer más a la comunidad que asume tales tareas. Para tales efectos se contabilizan y analizan artículos publicados acerca de asuntos probatorios en un grupo de revistas prestigiosas, teniendo en especial consideración las disciplinas de origen de sus autores, las materias tratadas y, en especial, las citas que en ellos se realizan.

Por cierto, con las citas y notas al pie se comunican diferentes cosas. De esta manera, las conclusiones que de ellas cabe extraer difícilmente resultarán precisas. Dice Anthony Grafton: "Para el inexperto, las notas al pie parecen sistemas profundamente arraigados, sólidos, firmes; para el entendido son auténticos hormigueros donde se desarrolla una actividad febril, constructiva y combativa. En Italia, por ejemplo, la nota al pie actúa por omisión tanto como por acción. El hecho de no mencionar a cierto estudioso o a cierta obra constituye una afirmación polémica, una damnatio memoriae que el círculo de interesados reconocerá y descifrará al instante [...]"15.

Los datos levantados dan pistas indirectas - pero no por ello poco relevantes-acerca de ciertas particularidades de la comunidad productora de textos de Derecho Probatorio y de su nivel de cohesión. Con ello, es posible esbozar un panorama preliminar acerca del nivel de desarrollo de la producción científica nacional y, a la vez, promover mejores formas de operar en este campo. Al no disponerse de un modelo consolidado acerca de cómo deben operar las disciplinas jurídicas ni tampoco de cómo de becho lo hacen, los

${ }^{14}$ Los beneficios de una mejor forma de producción y validación de los hechos, incluso se traduce en una mejor comprensión del derecho. Twining, W., "Taking Facts Seriously-Again”, en Twining, W., Rethinking Evidence, Cambridge University Press, Cambridge, 2a edición, 2006, pp. 418-419.

${ }^{15}$ Grafton, A., Los orígenes trágicos de la erudición, Fondo de Cultura Económica, México, 2015, p. 16. 
datos levantados no se confrontan con un grupo de control. En vista de lo señalado, investigaciones futuras podrían dar cuenta de que en otras disciplinas consolidadas se podrían presentar dinámicas de citación, también, sorprendentes.

\section{DATOS QUE CONTRIBUYEN A DIMENSIONAR EL PROBLEMA. Citas en escritos de Derecho Probatorio en revistas Chilenas INDEXADAS (2002-2014)}

\section{Algunas consideraciones acerca de la cobesión de quienes producen coetáneamente saberes respecto de un mismo objeto de estudio}

Los textos académicos dan pistas acerca de la cobertura y calidad de los saberes en un determinado campo. Así, por ejemplo, la disponibilidad de un número elevado de textos que abordan cuestiones de detalle constituye un indicador de la amplia cobertura de los saberes disponibles. A su vez, el uso de un lenguaje técnico que cuida la estrecha conexión de las distintas premisas de los argumentos planteados (no hay saltos) sin remitirse a cuestiones teóricas generales (salvo si se cuestiona lo que está en la base de la disciplina) refleja que los saberes disponibles son profundos. Un background abundante de saberes y a la vez profundo, presta gran utilidad para resolver exitosamente los enigmas que se presentan en la práctica cotidiana de los tribunales.

El ritmo de producción de nuevos conocimientos depende -a lo menos parcialmentedel nivel de cohesión de los autores de la respectiva disciplina. A mayor nivel de cohesión -y manteniéndose las demás variables inalteradas- se avanzará más rápidamente en la solución de los problemas de detalle, ganándose con ello en cobertura y profundidad ${ }^{16}$. Así, es útil distinguir entre comunidades de autores que tienen niveles de cohesión alto e intermedio y bajo (en este último escenario, incluso, es posible dudar si realmente se está en presencia de una disciplina $)^{17}$. La cohesión de un grupo de personas puede medirse, por ejemplo, por la participación en congresos de especialistas o por la publicación de libros homenaje cada vez que un miembro destacado cumple una cierta edad. También puede medirse por las citas bibliográficas o notas a pie de página. Solo esto último es lo que se considera aquí. En una comunidad altamente cohesionada debiese haber un alto porcentaje de citas recíprocas, es decir, distintos autores se hacen cargo de lo que entre ellos ya ha sido dicho, ya sea mediante referencias en el cuerpo de sus textos, o bien, con citas o notas a pie de página ${ }^{18}$. A su vez, en comunidades altamente cohesionadas debiese

${ }^{16}$ La observación la tomo, por cierto, de Thomas Kuhn, quien -como se sabe- refiere a las comunidades científicas, distinguiendo entre períodos de ciencia normal y períodos de crisis. Kuhn, Th., "La tensión esencial: tradición e innovación en la investigación científica”, en Kuhn, Th., La tensión esencial. Estudios selectos sobre la tradición y el cambio en el ámbito de la ciencia, FCE, Madrid, 1993 (2a reimp.), pp. $248-262$.

17 Ver Coloma, R., Las disciplinas jurídicas y su reinvención (en prensa).

18 Una investigación interesante y que aquí no se llevará a cabo sería el análisis de las referencias planteadas en congresos o en seminarios especializados. 
haber un elevado número de citas concordantes, esto es aquellas que aluden a textos comunes $^{19}$. Por supuesto, es posible que las citas recíprocas o concordantes involucren solo a un pequeño subgrupo que escribe respecto de la materia, resultando insuficiente para que se pueda hablar de una comunidad disciplinaria altamente cohesionada.

Cuando las citas recíprocas y/o concordantes se dan en un grupo reducido de autores, posiblemente se estará en presencia de redes de cooperación o de influencias. Esta forma de organización es bastante versátil y presta mucha utilidad para la generación de conocimientos. La pertenencia a una misma comunidad disciplinaria no constituye una condición para ser aceptado en ellas y la residencia en países distintos es vista a veces como una ventaja, pues amplía las perspectivas de análisis.

La cohesión será intermedia si las citas coinciden en un núcleo común de autores, no necesariamente nacionales. De lo que se trata es que ante una cierta clase de problemas, todos o la mayoría recurren a uno o más autores canónicos. Por cierto, la confluencia debiese darse respecto de un número relativamente elevado de textos citados e involucrar a un conjunto relevante de autores que citan. La cohesión será baja si no concurren las variables anteriores, esto es no hay citas recíprocas o estas son muy bajas, ni tampoco se produce un encuentro respecto de los textos canónicos en la disciplina. En el caso de comunidades altamente cohesionadas el conocimiento se concibe de manera incremental; en cambio en comunidades con escasa cohesión (no comunidades) el conocimiento es independiente, esto es no progresa de manera coordinada.

Tabla 1

NIVELES DE COHESIÓN DE LAS COMUNIDADES DE AUTORES EN UN MISMO CAMPO

\begin{tabular}{lcccc}
\hline \multicolumn{1}{c}{ Comunidad } & $\begin{array}{c}\text { Citas } \\
\text { recíprocas }\end{array}$ & Citas concordantes & $\begin{array}{c}\text { Citas } \\
\text { independientes }\end{array}$ & $\begin{array}{c}\text { Desarrollo del } \\
\text { conocimiento }\end{array}$ \\
\hline Altamente cohesionada & Frecuentes & Frecuentes & Ocasionales & Incremental \\
\hline $\begin{array}{l}\text { Medianamente } \\
\text { cohesionada }\end{array}$ & Ocasionales & Frecuentes & Ocasionales & Relacionado \\
\hline $\begin{array}{l}\text { No cohesionada } \\
\text { (No comunidad) }\end{array}$ & $\begin{array}{c}\text { Muy } \\
\text { infrecuentes }\end{array}$ & $\begin{array}{c}\text { Ocasionales/ } \\
\text { escasas }\end{array}$ & Frecuentes & Independiente \\
\hline
\end{tabular}

${ }^{19}$ Para hablar de citas concordantes debe existir una forma de uso de los textos que no difiera demasiado entre sí. Así, si un mismo grupo de textos es citado, es usado por algunos para profundizar ciertas tesis; y para dar cuenta de planteamientos erróneos por parte de otro, no cabría sostener que se trataría de una comunidad cohesionada. 


\section{Acerca de citas en revistas indexadas como espejo de la comunidad de autores}

Debido al soporte perdurable en que son presentados y su, muchas veces, cuidada estructura narrativa ${ }^{20}$, los textos escritos juegan un rol muy relevante para la optimización de los argumentos vertidos en el campo jurídico. La circunstancia aludida incide en que los autores suelen ser especialmente cuidadosos en las propuestas interpretativas, en la sistematización de las disposiciones, en la reconstrucción de las prácticas judiciales, y, en general, en los argumentos que en sus escritos se formulan, pues así evitarán tener que arrepentirse en el futuro.

Salvo circunstancias extraordinarias (textos fundantes), se espera que los nuevos textos incrementen los conocimientos acumulados. Así las cosas, los autores deben hacerse cargo de saberes aceptados hasta ese momento, ya sea usándolos en su favor, o bien refutándolos. No referir a los saberes consolidados expone a los nuevos textos al peligro de no ser tomados suficientemente en serio, pues las comunidades disciplinarias no gustan reinventar lo ya sabido. Las comunidades de trabajo serias se preocupan de dejar en claro que no todo vale y que es su función controlar lo que se produce. A su vez, lo que supera los filtros disciplinarios requiere ser potenciado. Al contrario, lo que fracasa está destinado al olvido, salvo en cuanto ilustre formas de operar que no han tenido éxito (y respecto de las cuales no conviene seguir intentándolo) ${ }^{21}$.

En lo que sigue se centrará la atención respecto de los artículos de Derecho Probatorio publicados entre el 2002 y 2014 en revistas chilenas WoS (ex ISI) y Scielo ${ }^{22}$. Esta categoría de revistas es, prima facie, una de las más considerablemente comprometidas con la producción de nuevos conocimientos (hay control de pares en la fase de aceptación ${ }^{23}$ e incentivos institucionales para publicar en ellas ${ }^{24}$ ), no existiendo dificultades de acceso a sus consumidores (disponibles gratuitamente en Internet). La función esperada de

${ }^{20}$ Los textos escritos hacen posible formas argumentativas más complejas que los discursos orales. Aquellos no requieren reiterar los puntos ya tratados, ni lidiar con auditorios fatigados que amenazan con perder la concentración. Los textos escritos permiten al autor afinar lo que dirá mediante revisiones de sucesivos borradores.

${ }^{21}$ Esto explica, por ejemplo, que una variable considerada en la evaluación de artículos presentados para la publicación en revistas sea la bibliografía (relevante y actualizada). La bibliografía también es considerada en los programas de asignaturas, en las discusiones de ponencias en congresos, etc.

${ }^{22}$ Las revistas revisadas han sido: a) Revista Chilena de Derecho (WoS); b) Revista de Estudios Constitucionales (algunos años ISI); c) Ius et Praxis; d) Política Criminal; e) Revista Chilena de Derecho Privado; f) Revista de Derecho (Coquimbo); g) Revista de Derecho (Valdivia); h) Revista de Derecho (Valparaíso); i) Revista de Estudios Histórico Jurídicos. Información relativa a cada una de estas revistas está disponible en www.scielo.cl

${ }^{23} \mathrm{El}$ arbitraje suele ser de doble ciego, lo que garantiza la existencia de un nivel básico de legitimación del texto publicado entre sus pares.

${ }^{24}$ La publicación en estas revistas aumenta la adjudicabilidad de proyectos de investigación (Conicyt), como asimismo, las universidades suelen ofrecer bonos en dinero por publicar en ellas. 
estos escritos es la de expandir el conocimiento y, a la vez, la de problematizar algunos puntos que se entiende que no han sido resueltos de una manera del todo satisfactoria ${ }^{25}$.

La elección del período 2002-2014, aparte de la circunstancia de que permite hacerse cargo de los artículos publicados desde el origen de la indexación de las revistas jurídicas nacionales, tiene el valor de que cubre una etapa en que las grandes reformas a los procedimientos en nuestro país comenzaban a implementarse ${ }^{26}$. Coincide además con una época en que una primera oleada de jóvenes doctores regresaba desde el extranjero. La elección de los textos recién indicados, por cierto, implica restricciones respecto del alcance de las conclusiones. Por una parte, quienes optan por publicar en las revistas indicadas han estado dispuestos a seguir tempranamente las políticas de investigación impulsadas por la Comisión Nacional de Ciencia y Tecnología y, a la vez, por sus propias universidades. De una rápida revisión de los nombres involucrados se observa que coinciden en una importante medida con lo que podríamos entender como una generación ${ }^{27}$ de académicos que en un elevado porcentaje estaba siendo contratada bajo un régimen de jornada completa en las universidades y que, a la vez, se venía incorporando a la vida académica en forma relativamente reciente ${ }^{28}$. De esta manera, quedan fuera varios procesalistas y otros dogmáticos ${ }^{29}$ que pertenecen a la generación anterior a la estudiada ${ }^{30}$, ya que su mayor compromiso investigativo está representado por la producción de libros ${ }^{31}$ y de capítulos en libros colectivos. Por cierto, sus textos muchas veces tienen una influencia considerable entre jueces y abogados.

${ }^{25}$ Los textos usados (consumidos) por los jueces, abogados y académicos con miras a apropiarse de saberes especializados son variados. Se cuentan, entre ellos, tratados, manuales, apuntes de clases y artículos de revista. Las funciones que es posible esperar de cada uno de ellos difieren, yendo desde una preocupación por difundir saberes estándar privilegiando el uso de un lenguaje asequible a una masa amplia de lectores, hasta la identificación de nuevos problemas o propuestas de soluciones originales, no escatimándose en el uso de un lenguaje abstruso que espanta a los amateurs.

${ }^{26}$ La reforma procesal penal comenzó en dos regiones del país el 16 de diciembre de 2000, llegando a cubrir la totalidad del país en junio de 2005.

${ }^{27}$ Entre nosotros quien más ha llamado la atención respecto de la idea de las generaciones -propuesta originariamente por Ortega y Gasset- es Edmundo Fuenzalida. Siguiendo sus criterios quienes escriben los textos analizados corresponderían fundamentalmente a la generación de 2001, esto es quienes cumplen 30 años en un período que cubre 7 años antes y 7 años después de esa fecha. Fuenzalida, E., "Investigación sociojurídica, cultura política y generaciones en Chile: un estudio exploratorio", en Anuario de Filosofía Jurídica y Social, 1998, pp. 342, 348 y ss.

${ }^{28}$ Agradezco observaciones en este punto de Daniela Accatino, Claudio Agüero, Mauricio Duce y Jean Pierre Matus.

${ }^{29}$ En el caso de los teóricos del derecho no hay representantes de las generaciones anteriores que se aboquen a asuntos probatorios.

${ }^{30} \mathrm{La}$ edad si bien es un elemento relevante para los efectos de determinar la pertenencia a una generación no es el único. Así, autores jóvenes que no han participado en la producción de artículos publicados en revistas indexadas quedan también fuera. Ellos suelen coincidir con jueces y abogados con una participación menor en el mundo universitario o que no cuentan con formación doctoral.

${ }^{31}$ Es indudable que para efectos de difusión del conocimiento los libros juegan un papel muy importante y aquí no serán considerados. En el caso del Derecho Procesal Penal ha sido particularmente evidente en los últimos 15 años, época en la que se han publicado libros de alto impacto que han tratado acerca de los problemas de regulación en asuntos de prueba y también han abordado cuestiones de técnicas de litigación. 
En la delimitación del corpus de textos y en la definición de los criterios de análisis se han tomado las siguientes decisiones ${ }^{32}$ :

a) Se asume una perspectiva restringida del Derecho Probatorio. Así, se establece como criterio de selección el uso de uno o más conceptos propios del Derecho Probatorio, ya sea en el título o en las palabras claves del artículo. Estos conceptos son actividad probatoria, admisibilidad, carga de la prueba, duda razonable, estándar de prueba, evidencia, hechos probados, peritos, prueba, razonamiento probatorio, sana crítica, testigos y valoración de la prueba ${ }^{33}$.

b) Se recaban pistas acerca de redes de colaboración en el Derecho Probatorio. Para ello se diferencia entre artículos unipersonales y en coautoría. Asimismo, se diferencia entre el tipo de citas que se realiza, distinguiendo entre citas genuinas, de compromiso y de erudición.

c) Se distinguen los asuntos abordados y, a la vez, las perspectivas de análisis asumidas en el texto. Es decir, se tiene en cuenta si su finalidad preferente es hacer precisiones conceptuales, propuestas de carácter normativo, levantamiento de datos empíricos o análisis metodológicos.

\section{Datos obtenidos}

El total de textos incorporados al corpus (base de datos) que a continuación se analiza es de 63 artículos. De ellos 13 corresponden a autores extranjeros, por lo que los cálculos consideran una base de 50 artículos. La clasificación que se lleva a cabo en la tabla inserta a continuación establece diferencias según i) la pertenencia disciplinaria de quienes escriben acerca de asuntos probatorios (las categorías utilizadas son las de Derecho Procesal Civil, Derecho Procesal Penal, Historia del Derecho, Teoría del Derecho, Derecho Laboral y Derecho Civil); ii) el número total de artículos que son generados por personas que pertenecen a cada una de estas disciplinas de origen; iii) el número de artículos que ha generado cada uno de los autores; y iv) los niveles de coautoría.

32 Según se señaló en nota inicial de este trabajo, la construcción de la base de datos estuvo a cargo de Victoria Martínez. Resultaron muy valiosas las sugerencias de Claudio Agüero respecto del diseño de esta, las que permitieron ir anticipando y evitando algunos problemas asociados a la selección de variables.

33 Por cierto, los conceptos probatorios son mucho más abundantes, por lo que los elegidos constituyen una muestra de aquellos que más se utilizan. Anderson, T., Schum, D., Twining, W., Analysis of Evidence, Cambridge University Press, Cambridge, $2^{\text {a }}$ ed., 2010 (Glosario), pp. 379-386. 
Tabla 2

PERTENENCIA A DISCIPLINAS Y COAUTORÍA

\begin{tabular}{|c|c|c|c|c|}
\hline & $\begin{array}{l}\text { Disciplina } \\
\text { principal }\end{array}$ & $\begin{array}{l}\text { Número total } \\
\text { de artículos }\end{array}$ & $\begin{array}{l}\text { Productividad individual. } \\
\text { Número de artículos / } \\
\text { cantidad de autor(es) que } \\
\text { han escrito ese número de } \\
\text { artículos }^{34}\end{array}$ & $\begin{array}{l}\text { Número de } \\
\text { artículos en } \\
\text { coautoría }\end{array}$ \\
\hline Procesalistas & $\begin{array}{c}\text { Derecho Procesal } \\
\text { Civil }\end{array}$ & 17 & $\begin{array}{l}6 / 1 \\
4 / 1 \\
1 / 7 \\
\end{array}$ & 4 \\
\hline \multirow{2}{*}{$\begin{array}{l}\text { Número total de } \\
\text { artículos (22) }\end{array}$} & $\begin{array}{l}\text { Derecho Procesal } \\
\text { Penal }^{35}\end{array}$ & 4 & $1 / 4$ & 0 \\
\hline & $\begin{array}{l}\text { Derecho Procesal } \\
\text { Constitucional }\end{array}$ & 1 & $1 / 1$ & 0 \\
\hline \multirow{2}{*}{$\begin{array}{l}\text { Teóricos } \\
\text { Número total de } \\
\text { artículos (15) }\end{array}$} & Teoría del Derecho & $14^{36}$ & $\begin{array}{l}8 / 1 \\
3 / 1 \\
1 / 3\end{array}$ & 4 \\
\hline & Historia del Derecho & 1 & $1 / 1$ & 0 \\
\hline \multirow[b]{3}{*}{$\begin{array}{l}\text { Dogmáticos (no- } \\
\text { procesalistas) }\end{array}$} & Derecho Laboral & 2 & $2 / 1$ & 1 \\
\hline & Derecho Penal & 2 & $1 / 2$ & 1 \\
\hline & Derecho Civil ${ }^{37}$ & 5 & $\begin{array}{l}2 / 1 \\
1 / 3\end{array}$ & 0 \\
\hline \multirow{3}{*}{$\begin{array}{l}\text { Número total de } \\
\text { artículos (13) }\end{array}$} & Derecho Comercial & 1 & $1 / 1$ & 0 \\
\hline & Derecho Canónico & 1 & $1 / 1$ & 0 \\
\hline & $\begin{array}{l}\text { Autor no se auto- } \\
\text { identifica con } \\
\text { ninguna disciplina }\end{array}$ & 2 & $1 / 2$ & 0 \\
\hline
\end{tabular}

\footnotetext{
${ }^{34}$ Para estos efectos se contabilizó solo al primer autor de cada artículo.

${ }^{35}$ Llama la atención la baja producción de los especialistas de Derecho Procesal Penal de esta clase de textos. Las razones pueden ser variadas, yendo desde la preocupación por la publicación de textos más extensos que den cuenta del nuevo sistema procesal penal (como ya se indicó, se han publicado varios libros), como también que varios de los asuntos de los que ellos debieran hacerse cargo han sido asumidos por teóricos del derecho o penalistas.

${ }^{36}$ En uno de los artículos se consideró la disciplina del segundo de los autores, ya que el primero es periodista.

${ }^{37}$ Uno de los autores puede ser considerado tanto civilista como procesalista (es profesor de Derecho Civil y Derecho Procesal Civil).
} 
Los asuntos respecto de los cuales se refieren los artículos analizados pueden agruparse en distintas categorías. Usaré las de problemas conceptuales, problemas normativos, problemas empíricos y problemas metodológicos. En los problemas conceptuales es factible incluir los intentos por depurar el significado de algunos conceptos con los cuales habitualmente se opera en el contexto del Derecho Probatorio. En los problemas normativos se incluyen trabajos que precisan los alcances de formulaciones normativas del sistema jurídico, o bien, que plantean casos que admiten solucionarse de determinada manera. En los problemas empíricos se incluyen trabajos que proveen datos acerca de cómo operan o cómo se ven a sí mismos los tribunales de justicia, los abogados, etc. En los problemas metodológicos se consideran trabajos que dan cuenta de herramientas para validar formas de producción de conocimientos. Por cierto hay textos que se preocupan de problemas que calzan con más de una categoría. A tales efectos, para no operar con más de una categoría, se tiene en consideración el porcentaje del texto que se dedica mayormente a uno de ellos, siendo ese el que será indicado en la tabla que sigue. Tomando como grupos que abordan distintos problemas los procesalistas, los dogmáticos sustantivos y los teóricos (incluye a los historiadores) se pueden extraer los siguientes datos.

Tabla 3

ENFOQUE DE LA INVESTIGACIÓN

\begin{tabular}{lccc}
\hline Enfoque de la investigación & Procesalistas & $\begin{array}{c}\text { Dogmáticos } \\
\text { sustantivos }^{38}\end{array}$ & Teóricos \\
\hline Conceptual & 0 & 1 & 11 \\
\hline Normativa & 22 & 12 & 1 \\
\hline Empírica & 0 & 0 & 2 \\
\hline Metodológica & 0 & 0 & 1 \\
\hline
\end{tabular}

La clase de problemas de los que se hacen cargo los textos académicos considera: a) Medios de prueba. Los asuntos refieren a cuestiones de admisibilidad, de iniciativa probatoria, del auto de prueba o de la resolución que recibe la causa a prueba y de algunas particularidades de los testigos, peritos y otros medios de prueba; b) Valoración de la prueba. El foco de atención está puesto en las inferencias que es válido realizar a partir de la prueba rendida. Así, por ejemplo, lo concerniente a la sana crítica se sitúa en esta categoría. Los problemas de la suficiencia de la motivación de la sentencia también se consideran aquí; c) Estándar de prueba y carga de la prueba; d) Otros. Aquí se consideran

\footnotetext{
${ }^{38}$ En esta categoría se incluyen los autores que no se autoidentifican con ninguna disciplina.
} 
textos que tratan de cultura, ética, etcétera, que no entran claramente en las categorías anteriores.

Tabla 4

PROBLEMAS PREFERENTEMENTE ABORDADOS

EN LA INVESTIGACIÓN

\begin{tabular}{lccc}
\hline Problemas abordados & $\begin{array}{c}\text { Procesalistas } \\
(\%)\end{array}$ & $\begin{array}{c}\text { Dogmáticos } \\
\text { sustantivos (\%) }\end{array}$ & $\begin{array}{c}\text { Teóricos } \\
\text { e historiadores (\%) }\end{array}$ \\
\hline Medios de prueba & 64 & 0 & 24 \\
\hline Valoración de la prueba & 23 & 67 & 38 \\
\hline Estándar y carga de prueba & 9 & 26 & 38 \\
\hline Otros & 4 & 7 & 0 \\
\hline
\end{tabular}

Las tablas que siguen se hacen cargo de las prácticas de citación tanto a un nivel micro como macro. Respecto de lo primero (tabla 5), se seleccionan seis artículos ${ }^{39}$ y se contabilizan las citas realizadas según autor ${ }^{40}$.

Las tres tablas que a continuación se incorporan (nivel macro) dan cuenta de la clase de citas que realizan los procesalistas, los dogmáticos sustantivos y los teóricos. Se diferencia entre citas genuinas, de erudición o de compromiso. Asimismo, se considera la adscripción disciplinaria del autor citado, en el caso de que sean nacionales; en el caso de autores citados extranjeros no se hace dicha diferenciación. Se incluye, además, una fila para dar cuenta de textos cuya autoría es de no abogados y que tampoco escriben acerca de asuntos de derecho.

39 Los textos seleccionados corresponden a los artículos más recientes de los autores con mayor productividad en cada área.

${ }^{40}$ El tamaño de la muestra es pequeño y no tiene pretensiones de representatividad. Las expectativas apuntan solo a ilustrar la clase de autores cuya citación es preferente. 


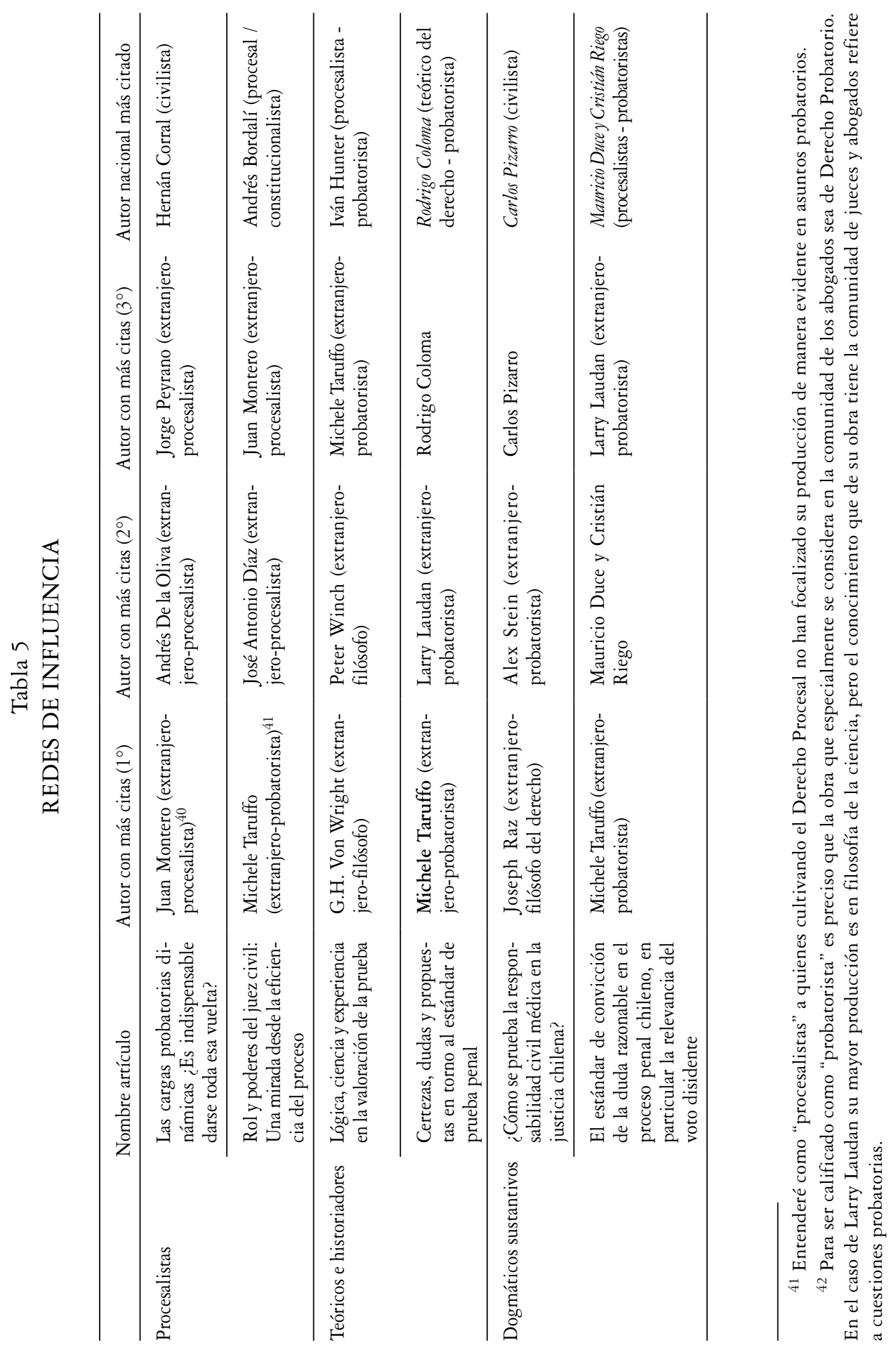


Entenderé por citas genuinas aquellas que contribuyen a fortalecer las ideas planteadas en el texto. Los indicadores de que se trata de citas genuinas son: a) Se cita directamente el texto; b) Se problematiza el texto citado; c) Se adopta una posición respecto del texto; d) El punto de vista del autor citado constituye un presupuesto del análisis que se realiza; e) Se hace cargo de la idea del autor; y f) Se hace referencia a una frase o concepto utilizado por el autor o su obra. En todos los artículos se contabilizaron 826 citas genuinas, que representan el 38\% del total. Entenderé por citas de erudición aquellas que comunican que el autor ha tenido a la vista un background amplio de lecturas y que lo que en los textos citados se dice puede ser tenido como un trasfondo de las ideas planteadas. Los indicadores de que se trata de citas de erudición son: a) Se utiliza la expresión véase o confrontar (cfr.) con miras a que el lector pueda obtener más información o profundizar en un asunto; b) Se alude a una obra o autor como el más importante o quién ha estudiado con mayor profundidad un tema específico; c) Se cita como respaldo de información enunciada en el texto. En todos los artículos se contabilizaron 1.083 citas de erudición, que representan el 50\% del total. Entenderé por citas de compromiso aquellas que son utilizadas para fortalecer redes académicas o, en ciertos casos, para no correr riesgos ante los arbitrajes a los que se someterá el texto. Los indicadores de que se trata de citas de compromiso son: a) No se hace cargo de la idea del autor; b) Se alude genéricamente a la doctrina; c) Se indica "véase", "a modo de ejemplo", o "entre otros" (citas sustituibles); d) Se mencionan para dar sustento a opiniones generales o a ideas de conocimiento común en la disciplina; e) Se cita para corroborar información de público conocimiento, que no necesita respaldo. En todos los artículos se contabilizaron 266 citas de compromiso, que representan el 12\% del total.

Tabla 6

PRÁCTICAS DE CITACIÓN DE LOS PROCESALISTAS

\begin{tabular}{lcccc}
\hline \multicolumn{1}{c}{ Artículos de procesalistas } & Citas genuinas & $\begin{array}{c}\text { Citas de } \\
\text { erudición }\end{array}$ & $\begin{array}{c}\text { Citas de } \\
\text { compromiso }\end{array}$ & Total \\
\hline $\begin{array}{l}\text { A sí mismo o a otros procesa- } \\
\text { listas chilenos }\end{array}$ & $52(15 \%)$ & $108(21 \%)$ & $38(25 \%)$ & $198(19.3 \%)$ \\
\hline A no procesalistas chilenos & $22(6 \%)$ & $36(7 \%)$ & $9(6 \%)$ & $67(6.5 \%)$ \\
\hline A autores extranjeros & $282(79 \%)$ & $368(71.6 \%)$ & $107(69 \%)$ & $757(74 \%)$ \\
\hline A no abogados & $0(0 \%)$ & $2(0.4 \%)$ & $0(0 \%)$ & $2(0.2 \%)$ \\
\hline Total & $356(100 \%)$ & $514(100 \%)$ & $154(100 \%)$ & $1.024(100 \%)$ \\
\hline
\end{tabular}


Tabla 7

PRÁCTICAS DE CITACIÓN DE LOS DOGMÁTICOS SUSTANTIVOS

\begin{tabular}{lcccc}
\hline \multicolumn{1}{c}{$\begin{array}{c}\text { Artículos de dogmáticos } \\
\text { sustantivos }\end{array}$} & $\begin{array}{c}\text { Citas } \\
\text { genuinas }\end{array}$ & $\begin{array}{c}\text { Citas de } \\
\text { erudición }\end{array}$ & $\begin{array}{c}\text { Citas de } \\
\text { compromiso }\end{array}$ & Total \\
\hline $\begin{array}{l}\text { A sí mismo o a otros dogmáticos } \\
\text { sustantivos }\end{array}$ & $13(6.5 \%)$ & $28(14 \%)$ & $0(0 \%)$ & $41(9 \%)$ \\
\hline A no dogmáticos sustantivos & $20(10 \%)$ & $22(11 \%)$ & $22(71 \%)$ & $64(15 \%)$ \\
\hline A autores extranjeros & $169(83 \%)$ & $146(73 \%)$ & $9(29 \%)$ & $324(75 \%)$ \\
\hline A no abogados & $1(0.5 \%)$ & $4(2 \%)$ & $0(0 \%)$ & $5(1 \%)$ \\
\hline Total & $203(100 \%)$ & $200(100 \%)$ & $31(100 \%)$ & $434(100 \%)$ \\
\hline
\end{tabular}

Tabla 8

PRÁCTICAS DE CITACIÓN DE LOS TEÓRICOS DEL DERECHO

\begin{tabular}{lcccc}
\hline \multicolumn{1}{c}{$\begin{array}{c}\text { Artículos de teóricos del } \\
\text { derecho }\end{array}$} & $\begin{array}{c}\text { Citas } \\
\text { genuinas }\end{array}$ & $\begin{array}{c}\text { Citas de } \\
\text { erudición }\end{array}$ & $\begin{array}{c}\text { Citas de } \\
\text { compromiso }\end{array}$ & Total \\
\hline $\begin{array}{l}\text { A sí mismo o a otros teóricos } \\
\text { chilenos }\end{array}$ & $11(5 \%)$ & $25(8 \%)$ & $8(10 \%)$ & $44(7 \%)$ \\
\hline A los no-teóricos chilenos & $24(10 \%)$ & $38(12 \%)$ & $11(14 \%)$ & $73(12 \%)$ \\
\hline A autores extranjeros & $148(62 \%)$ & $174(57 \%)$ & $54(67 \%)$ & $376(60 \%)$ \\
\hline A no abogados & $55(23 \%)$ & $70(23 \%)$ & $7(9 \%)$ & $132(21 \%)$ \\
\hline Total & $238(100 \%)$ & $307(100 \%)$ & $80(100 \%)$ & $625(100 \%)$ \\
\hline
\end{tabular}

\section{Discusión. Algunas particularidades de la comunidad QUE ESTUDIA ASUNTOS PROBATORIOS}

En la tradición del Common Law se cuenta con una disciplina (rama del derecho) específica que se hace cargo del campo de los problemas probatorios. Dicha práctica favorece un análisis sofisticado apto para dar cuenta de las particularidades de los recursos que se busca optimizar. En nuestra tradición aquello no ocurre aun cuando podría entenderse que, desde hace algún tiempo, se han dado pasos en esa dirección. Los textos que forman parte del corpus analizado -y, sobre todo las prácticas de citación- son indicativos de que podría estar constituyéndose una nueva comunidad. Sin embargo, aquello sería una conclusión muy apresurada. 
De los datos recogidos es posible extraer características de los saberes probatorios y de la comunidad de autores que los ha venido produciendo. Llamaré la atención en solo dos de ellas: la primera refiere a que la aludida comunidad cuenta con un nivel mediobajo de cohesión (ver tabla 1); y la segunda, que el enfoque adoptado en los textos se encuentra notoriamente determinado por la disciplina de origen de sus autores.

La señal más clara de la escasa cohesión que habría entre los autores se extrae del elevado porcentaje de citación a autores extranjeros (74\%), en comparación con lo que ocurre respecto de autores nacionales. El fenómeno se presenta tanto a nivel macro (tablas 6, 7 y 8) como a nivel micro (tabla 5). Tales prácticas de citación admiten interpretarse en términos de que lo que se produce a nivel local es muy insuficiente ${ }^{43}$ (da cuenta de una cantidad reducida de problemas) o carece de credenciales suficiente como para ser objeto de discusión (no satisface estándares cualitativos o de relevancia). Lo problemático de dicha constatación -sobre todo la segunda- es que el conocimiento producido a nivel local no estaría en condiciones de asumir una lógica incremental, esto es, cada uno puede comenzar nuevamente a discutir de un determinado asunto sin hacerse cargo de lo precedentemente dicho. La existencia de una comunidad altamente cohesionada no requiere andar repitiendo las $\operatorname{cosas}^{44}$ y puede situarse rápidamente en problemas de alto interés para los lectores. Esto le permite resolver varias cuestiones de detalle, lo que influye en una mejor disposición de los jueces y abogados a los efectos de dar un mayor uso a los textos a nivel de litigación.

Las señales que, en cambio, apuntan hacia un nivel medio de cohesión refieren a la existencia de citas concordantes ${ }^{45}$ (en la tabla 5 aparece en cuatro ocasiones Michele Taruffo $^{46}$, y en dos, tanto Larry Laudan como Juan Montero). Asimismo, hay niveles de coautoría que no serían bajos tomando en cuenta las prácticas de otras comunidades abocadas al estudio de problemas jurídicos (tabla 2). La coautoría refleja la existencia

${ }^{43}$ Los recientes cambios en los procedimientos podrían explicar la escasez de textos nacionales a los que se hace referencia en las publicaciones estudiadas. Ante esta posibilidad se analizaron los datos recogidos únicamente en el período 2011-2014. Los porcentajes observados permanecieron prácticamente inalterados. Solo hubo una reducción de autores no abogados en el caso de los teóricos del derecho.

${ }^{44}$ El asunto, por cierto, no atañe solo a juristas. André Gide en Le traité du Narcisse dice: "Todo ha sido dicho antes, pero dado que nadie escucha, tenemos que regresar y empezarlo todo otra vez". Tomado de Gross, J., The Oxford Book of Aphorisms, Oxford University Press, Oxford (reimpresión), 2010, p. 1.

${ }^{45}$ Wallerstein destaca que las disciplinas pueden ser entendidas como una cultura: "Habitualmente, los académicos que dicen pertenecer a un grupo disciplinar comparten con los otros miembros del grupo experiencias y contactos: han leído los mismos textos "clásicos"; participan de los mismos debates tradicionales, que a menudo son distintos de los de las disciplinas lindantes, tienen un mismo estilo y reciben recompensas por ello. Y si bien esa cultura puede modificarse con el tiempo -y de hecho así sucede-, si se hace un corte temporal de un período determinado, se aprecian modos de presentación que son valorados por los miembros de una disciplina y no por los de otra". Wallerstein, I., "La antropología, la sociología y otras disciplinas dudosas", en Wallerstein, I., Las incertidumbres del saber, Gedisa, Barcelona, 2005, pp. 141-142.

${ }^{46}$ El caso de Michele Taruffo tiene, además, una particularidad. Sus textos son citados en 30 de los 50 artículos analizados. Es posible que el éxito se deba a su capacidad para operar en distintos ámbitos: Derecho Procesal italiano y estadounidense, epistemología, filosofía moral, etc. El título de un reciente congreso realizado en Girona da cuenta de ello: "Un jurista pluridisciplinar: en torno al pensamiento de Michele Taruffo". 
de, al menos, pequeñas redes de colaboración al suponer un alto nivel de concordancia entre quienes redactan un texto ${ }^{47}$.

Respecto del enfoque de la investigación, en la tabla 3 se advierte claramente una división de intereses entre los procesalistas y dogmáticos sustantivos, por una parte, y los teóricos, por la otra. Por cierto, aquello no debiera provocar mayores sorpresas. El problema es que aquello podría reflejar que el desarrollo va por carriles paralelos que no se alimentan recíprocamente. Esto ocurriría si los procesalistas y los dogmáticos sustantivos no usan las precisiones conceptuales de los teóricos y, a la vez, estos últimos no prestan atención a las interpretaciones de los primeros. A lo anterior se suma que en la tabla 4 se da cuenta que la disciplina de origen determina de manera relevante el tipo de problemas de los que cada autor se hace cargo ${ }^{48}$. Lo advertido, sin embargo, requiere ser matizado, pues no resultan infrecuentes las citas a miembros de otras comunidades de origen. En las tablas 6, 7 y 8 se advierte que los procesalistas no citan solo a procesalistas, lo que también aplica a dogmáticos sustantivos y a teóricos.

\section{El Derecho Probatorio como un campo abierto y escasamente explorado}

Los estudios acerca de problemas de prueba no se han hecho cargo de ellos en toda su complejidad. A diferencia de lo que ocurre en varias disciplinas dogmáticas, un "Derecho Probatorio" excesivamente centrado en la identificación e interpretación de disposiciones normativas, resulta impotente para operar satisfactoriamente en el vasto campo de las decisiones factuales ${ }^{49}$. Lo que abogados y jueces buscan en los textos de los especialistas es un set de herramientas útiles para solucionar problemas prácticos respecto de los cuales las disposiciones del legislador suelen decir bastante poco ${ }^{50}$. Como es de esperar, cuando los espacios quedan escasamente cubiertos por disposiciones normativas, se generan buenas

${ }^{47}$ En nuestro país hay, además, experiencias de diálogo entre autores exponentes de distintas disciplinas jurídicas. Este es el caso de los Talleres de Estudios Probatorios que organizan en conjunto las universidades Alberto Hurtado y Diego Portales. La iniciativa lleva 5 versiones contándose entre sus participantes jueces, abogados y académicos con filiación en el Derecho Procesal Penal, Derecho Procesal Civil y Filosofía del Derecho. En el ámbito de la investigación puede hacerse notar, a modo de ejemplo, que en el proyecto al que se adscribe este trabajo se encuentra conformado por tres teóricos del derecho y tres procesalistas. Por cierto, estos ejemplos son absolutamente insuficientes como para sostener que estamos en presencia de una comunidad que dialoga con cierta regularidad.

${ }^{48}$ Los procesalistas se centran preferentemente en lo que concierne a los medios de prueba. Los dogmáticos, en cambio, están más preocupados de los problemas de valoración. En el caso de los teóricos los estudios están más distribuidos, prestándole algo menos de atención a los medios de prueba.

${ }^{49}$ Un amplio universo de problemas interpretativos de los cuales se hacen cargo los abogados que operan en distintas ramas del derecho se enriquecen al utilizarse saberes externos (por ejemplo, los penalistas cuando abordan el problema de la "locura" o "demencia"). En el caso del Derecho Probatorio aquello es mucho más frecuente.

${ }^{50} \mathrm{La}$ literatura es abundante. Un bastión en la defensa de la prueba libre fue Jeremy Bentham. Bentham, J., Tratado de las pruebas judiciales, E.J.E.A, Buenos Aires, Vols. I y II, 1971. Ver también Cohen, J., "Freedom of Proof", en Twining, W., Stein, A., Evidence and Proof, New York University Press, New York, pp. 3-23. En contra, ver Stein, A., "Contra la prueba libre", en Revista de Derecho (Valdivia), vol. XXVI, $\mathrm{N}^{\circ} 2$, 2013, pp. 245-261. 
razones para buscar respuestas fuera de ellas. En ese escenario, las respuestas de adentro ganan en reconocimiento en la medida que no entren en tensión con otras disponibles afuera, las que se vislumbran útiles para operar en los procesos judiciales ${ }^{51}$. Así, en los textos debe prestarse atención a saberes y métodos de otras disciplinas ${ }^{52}$.

Roger Park y Michael Saks, a partir del análisis de 10 revistas jurídicas norteamericanas reconocidamente influyentes, advierten un importante giro en el tipo de artículos sobre problemas probatorios que en ellas se publican. A comienzos del siglo XX, el 93\% de los artículos eran doctrinales, esto es textos que describen y sintetizan disposiciones jurídicas, o bien que sugieren mejoras o reformas a la legislación (los saberes de otras disciplinas cumplen una función puramente auxiliar). A mediados de siglo, la cifra de textos doctrinales baja a $79 \%$, para seguir reduciéndose a fines de la centuria llegando a solo el $20 \% 53$.

Históricamente han sido los procesalistas quienes se han hecho cargo de un elevado número de problemas de prueba. Respecto de otros, han preferido guardar prudente distancia. Así las cosas, otras disciplinas jurídicas han ido asumiendo estos últimos como parte de su objeto de estudio ${ }^{54}$. Los asuntos respecto de los cuales los procesalistas se han replegado corresponden, por una parte, a cuestiones que son tratadas en los códigos de otras disciplinas ${ }^{55} \mathrm{y}$, por la otra, a asuntos en que la normativa vigente da indicaciones muy menores ${ }^{56}$.

El fenómeno de la repartición del campo de los asuntos probatorios no es un fenómeno exclusivamente local ${ }^{57}$, ni se reduce a cultores de disciplinas solo jurídicas. ${ }^{58}$

51 Para la legitimidad de las decisiones judiciales no resulta indiferente la existencia de relatos alternativos de los historiadores, de los economistas, de los físicos o de los periodistas.

${ }^{52}$ De otra manera resulta muy difícil, por ejemplo, hacerse cargo de lo que dicen los peritos o resolver satisfactoriamente problemas de credibilidad de testigos.

${ }^{53}$ Park, R., Saks, M., "Evidence Scholarship Reconsidered: Results of the Interdisciplinary Turn”, en 47 Boston College Law Review, 2006, p. 952.

${ }^{54}$ Por supuesto que hay también asuntos que los procesalistas tratan con bastante propiedad y respecto de los cuales, los externos no se inmiscuyen.

55 El caso más vistoso refiere al Código Civil que en uno de sus títulos trata de la "prueba de las obligaciones". Es ilustrativo, además, que en los textos estudiados los procesalistas no se hayan ocupado, por ejemplo, de los problemas que atañen al proceso laboral, tal vez porque las disposiciones respectivas están en el Código del Trabajo.

${ }^{56}$ Aquello se produce claramente respecto de la valoración de la prueba conforme a la sana crítica o en los estándares de prueba, donde el legislador es muy parco. En cuestiones de admisibilidad, el legislador es mucho más pródigo y, en consecuencia, los procesalistas se sienten más a gusto.

${ }^{57}$ En España, por ejemplo, es indudable la influencia de un grupo de filósofos del derecho (Perfecto Andrés Ibáñez, Jordi Ferrer, Marina Gascón, Daniel González Lagier y algunos otros) en lo que refiere a varios asuntos de Derecho Probatorio.

${ }^{58}$ Es interesante, por ejemplo, que Larry Laudan -un prestigioso filósofo de la ciencia-se haya convertido en un referente casi obligado en asuntos de naturaleza conceptual que atañen al estándar de prueba. Otra figura influyente desde el campo de la epistemología es Susan Haack, quien se ha ocupado principalmente del rol que es posible asignar a la verdad en asuntos de prueba, en el razonamiento probatorio y en la prueba 
El Derecho Procesal se muestra llano a que otros hablen acerca de asuntos de prueba, especialmente si se trata de problemas de naturaleza conceptual ${ }^{59} \mathrm{y}$, en una menor medida, si se abordan problemas de filosofía moral o política ${ }^{60}$. Las otras disciplinas dogmáticas no están preocupadas solo de lo que se dice en los códigos de los cuales están a cargo, lo que pareciera ser una señal de que, al menos algunos de sus cultores, no se sienten muy a gusto con la distinción fuerte -que suele darse entre nosotros- entre asuntos sustantivos y asuntos adjetivos ${ }^{61}$. Pese a ello, no parecen incomodarse con otros participantes, tal vez porque asumir todos los problemas probatorios les parece una carga demasiado pesada. Los teóricos, por su parte, eligen los problemas de los cuales se harán cargo sin que constituya un factor de preocupación de que los mismos sean abordados también desde otras perspectivas, ni que tampoco haya asuntos probatorios de los que ningún otro teórico se preocupe.

La existencia de un espacio común con diversidad de participantes no es en absoluto preocupante. El Derecho Probatorio, en cuanto objeto de estudio, se empobrece si se le entiende como un repositorio de proposiciones acerca de disposiciones normativas ${ }^{62}$. La dogmática de la prueba no está en condiciones de neutralizar a la epistemología y a la filosofía moral provenientes "desde fuera" del ámbito jurídico ${ }^{63}$. Ambas parecieran encontrarse en buenas condiciones para producir respuestas útiles para la decisión de

científica. Una buena y reciente selección de sus artículos puede verse en Haack, S., Evidence Matters: Science, Proof, and Truth in the Law, Cambridge University Press, New York, 2014. Por su parte, para los problemas de credibilidad suele ser mucho más interesante lo que dicen los psicólogos que lo que dicen los juristas.

${ }^{59}$ Hay libros que en su título incorporan la locución "epistemología jurídica”, lo mismo que congresos de especialistas. Hablar de "epistemología jurídica" es ambiguo, pues también da cuenta de problemas de conocimiento del derecho. Más allá de estos problemas, aquella comunica en el Derecho Probatorio cercanía con la epistemología general, en términos de contribuir a la reducción (control) de errores. Cfr. Laudan, L., Verdad, error y proceso penal. Un ensayo sobre epistemología jurídica, Marcial Pons, Madrid, 2013, pp. 23-25.

${ }^{60}$ La buena acogida de otros saberes se ha visto potenciada con el retroceso de la prueba tasada en la fase de valoración de la prueba (unida a una mayor reticencia de los códigos a considerar inadmisibles ciertas pruebas).

${ }^{61}$ Ya no es tan infrecuente que los dogmáticos en cuestiones sustantivas hagan también cursos en cuestiones adjetivas. Esto se da especialmente entre los penalistas que gustan hacerse cargo de cuestiones propias del proceso penal (como se sabe, la división entre lo sustantivo y lo adjetivo es mucho más tenue en otras tradiciones).

${ }^{62}$ Entre nosotros no se ha producido una discusión intensa respecto de cuánto deben hacerse depender las soluciones a los problemas probatorios de lo que el legislador disponga y cuánto de la epistemología general. En la tradición del Common Law el asunto ha sido largamente, sobre todo, a partir de las propuestas de Jeremy Bentham (ver Twining, W., Theories of Evidence: Bentham \& Wigmore, Weidenfeld \& Nicolson, London, 1985, pp. 1-18) a quien se ha erigido como un paladín de la perspectiva de la desregulación y de la aplicación directa de criterios de validación epistémicos. El asunto, por cierto, permanece abierto (ver Bayón, J. C., "Epistemología, moral y prueba de los hechos: hacia un enfoque no benthamiano", en Analisi e Diritto, 2008, pp. 15-34).

Excluyendo posiciones que habrían de situarse en los extremos, la sola existencia de la discusión es indicativa de la utilidad de un diálogo fluido entre puntos de vista con un compromiso primordial con las reglas jurídicas, o bien, con la epistemología general.

${ }^{63}$ Entre los anglosajones resulta más frecuente hacer una distinción entre problemas de reglas y problemas de lógica. El giro advertido en nuestro país implica prestar mayor atención a los problemas lógicos, los que son comunes a distintos espacios de la vida donde se opera con pruebas. 
los hechos en el proceso. La situación es distinta a lo que ocurre en campos en los que se dispone de construcciones teóricas que neutralizan lo que se dice, por ejemplo, desde la filosofía moral o la economía. El background de saberes y de métodos que potencialmente llegarán a pertenecer al Derecho Probatorio debiese incluir también proposiciones que para su justificación requieran enfrentar un proceso de validación cercano al esperable para las disciplinas científicas. El auge del sistema de la sana crítica, por ejemplo, implica que para enfrentar los problemas de valoración de la prueba sea menester explorar mecanismos que exceden con creces lo intuitivo o lo que es de "sentido común"64. Así, se busca establecer cortapisas a la discrecionalidad judicial (e indirectamente, a los argumentos de las partes) cuando lo que se dice entra en tensión con conocimientos científicamente afianzados, máximas de la experiencia y principios de la lógica ${ }^{65}$. En un escenario de esa naturaleza la epistemología, entendida como una disciplina que establece condiciones de validación a los conocimientos -o a las actitudes doxásticas ${ }^{66}$ - que se van produciendo, aparece como muy útil. De la epistemología podemos tener pistas acerca de qué es lo que cuenta como conocimiento científicamente afianzado, cuál es el valor que podemos asignar a las experiencias previas (o al sentido común) en espacios en los que la ciencia poco puede decirnos e, incluso, acerca de la lógica que es posible emplear en contextos como el de los procesos judiciales.

Hay problemas difíciles de resolver al momento de hacer transferencias de contextos. Una epistemología que se hace cargo de lo que ocurre en el seno de comunidades científicas, o en la vida diaria de las personas, presenta algunas particularidades que es preciso observar cuidadosamente, cuando se opera en sede judicial. Aquello supone un desafío del que deben hacerse cargo los autores. Así, debe haber suficiente claridad respecto de los propósitos y de los conceptos, si no se quiere permanecer en un lugar que a algunos pudiera asemejarse a la Torre de Babel. Por cierto, aquello excede de lo pretendido en este texto, pero vale la pena estudiarlo.

\section{Conclusiones y proyecciones PARA El Derecho Probatorio}

a) Con miras a explicar el bajo uso de los textos de Derecho Probatorio -por parte de jueces y abogados- se analizaron prácticas de citación y, en menor medida, se identificaron temáticas y enfoques asumidos en artículos publicados en revistas

${ }^{64}$ Entre nosotros no se han producido discusiones respecto de las potencialidades que representa, por ejemplo, el Teorema de Bayes. Al respecto se ha producido una abundante literatura, principalmente en la tradición anglosajona.

65 Coloma, R., Agüero, C., "Lógica, ciencia y experiencia en la valoración de la prueba", en Revista Chilena de Derecho, vol. 41, N², 2014, pp. 673-703.

${ }^{66}$ Ver Goldman, A., "A guide to Social Epistemology”, en Goldman, A., Social Epistemology. Essential Readings, Oxford University Press, Oxford, 2011, pp. 11-14. 
indexadas. Se observó que la comunidad ${ }^{67}$ a cargo de la producción de saberes probatorios no concibe su trabajo como una empresa suficientemente colaborativa: sus aportes no son incrementales. Entre quienes abordan asuntos de prueba se advirtió una multiplicidad de perspectivas que en razón de bajos niveles de intercambio conspira en contra de la optimización de los recursos disponibles.

b) Algunos procesalistas tienen dificultades (o escaso interés) en asuntos de valoración de la prueba en contextos distintos al de la prueba tasada. Por su parte, quienes no son procesalistas (especialmente los teóricos) tienen dificultades (o escaso interés) por esclarecer lo que dicen los códigos (admisibilidad, determinación de los hechos a probar, etcétera). En asuntos en que el legislador ha tomado posición mediante la dictación de reglas, la dogmática (de los procesalistas) provee de herramientas, por regla general, adecuadas. Para dirimir, en cambio, la manera en que se debe operar en asuntos más indeterminados, la filosofía moral (que resulta familiar a los teóricos del derecho) y la epistemología (cuyo dominio no se asocia a una disciplina específica) resultan de mucha utilidad.

El encuentro de la dogmática con la epistemología y la filosofía moral debiese producir efectos muy positivos para la superación de los problemas anunciados ${ }^{68}$. Sin embargo, hasta el momento, los saberes que se generan en cada uno de esos campos no se conectan de una manera suficientemente estrecha, como para que lo que se diga en uno impacte sobre lo que se dice en los otros. Tales mundos aparecen como distantes, cuestión que podría ser consecuencia de la falta de propósitos comunes respecto de lo que cabe esperar del Derecho Probatorio (¿es posible una teoría general de la prueba?). Los consumidores de textos que se enfrentan en la cotidianeidad a problemas prácticos estarán mucho más dispuestos a usar los recursos optimizados que ofrecen los textos, en la medida que se resuelvan los mencionados desencuentros.

c) Dos puntos no tratados en este texto y que merecen atención en el marco de lo que debiera hacerse para superar algunas de las insuficiencias del Derecho Probatorio, refieren a la carencia de un modelo conceptual y a los déficit en la formación de los futuros abogados. El vocabulario del Derecho Probatorio requiere precisiones, lo mismo que urgen modelos que apoyen la tarea judicial de adscribir conductas normativamente relevantes a los partícipes en los juicios ${ }^{69}$.

${ }^{67}$ En su mayoría académicos de jornada completa o media jornada con formación doctoral y menores de 50 años.

${ }^{68}$ Bayón, J. C., "Epistemología, moral y prueba de los hechos: hacia un enfoque no benthamiano", en Analisi e Diritto, 2008, pp. 15-34.

${ }^{69}$ Cuenta Larry Laudan, que su interés por lo que ocurría en el derecho fue originado por perplejidades acerca de lo que los tribunales querían decir al invocar la duda razonable, la que fue ampliándose a varias otras nociones utilizadas por abogados y académicos. Laudan, L., Verdad, error y proceso penal. Un ensayo sobre epistemología jurídica, Marcial Pons, Madrid, 2013, pp. 13-14. 
Entre los conceptos que requieren con urgencia de consensos interpretativos básicos (más allá de los legítimos disensos) pueden mencionarse, entre otros, "relevancia”, "peso probatorio", "presunciones”, "estándar de prueba”, "carga de la prueba”, "hecho probado” y "medios de prueba”. Todos ellos además de una depuración conceptual requieren de la construcción de una estructura de relaciones recíprocas que permita visualizar qué implicaciones representa cada concepto respecto de los restantes ${ }^{70}$. La anunciada red conceptual atenuará los problemas de contradicciones, redundancias y lagunas que es usual se produzcan en las disposiciones (del legislador) y en las proposiciones normativas (de los juristas). Asimismo, facilitará la producción de explicaciones ante dudas frecuentes que son planteadas desde dentro y fuera del espacio de lo jurídico. Por último, contribuirá a fortalecer al Derecho Probatorio como disciplina responsable de la producción de conocimientos en el contexto de la adjudicación, capaz de enriquecerse con los aportes desde fuera del sistema jurídicos, pero a la vez, dispuesta a establecer barreras frente propuestas que no se hagan cargo suficientemente de las exigencias esperables respecto de los hechos que alcanzan el estatus de probados en los procesos judiciales.

La educación de los futuros abogados debe hacerse cargo de los problemas de prueba. Aquello requiere ciertos cambios en la concepción que se tiene del proceso formativo. Hasta hace poco tiempo las facultades de derecho han eludido el problema sin grandes dificultades. Es previsible que en el corto y mediano plazo aquello no se sostenga. Un desafío para la comunidad de autores que abordan los problemas de prueba es la creación de materiales que resulten adecuados para enfrentar dicho desafío. Los nuevos textos, entre otras cosas, deberán hacerse cargo del entorno donde se producen los problemas de prueba. Los aprendizajes deberán ser satisfactorios en un escenario en que la autonomía disciplinar no permite hacer oídos sordos a lo que se produce "desde fuera".

\section{BIBLIOGRAFÍA}

Alcalá-Zamora, N., Estudios de Derecho Probatorio, Universidad de Concepción, Concepción, 1965. Anderson, T., Schum, D., Twining, W., Analysis of Evidence, Cambridge University Press, Cambridge, $2^{\mathrm{a}}$ ed., 2010.

BAYón, J. C., "Epistemología, moral y prueba de los hechos: hacia un enfoque no benthamiano", en Analisi e Diritto, 2008.

Bentham, J., Tratado de las pruebas judiciales, E.J.E.A, Buenos Aires, Vols. I y II, 1971.

Bourdieu, P., "Elementos para una sociología del campo jurídico", en Bourdieu, P., Teubner, G.. La fuerza del derecho, Universidad de los Andes y otras, Bogotá, 2000.

Cohen, J., "Freedom of Proof", en Twining, W., Stein, A., Evidence and Proof, New York University Press, New York, pp. 3-23.

Coloma, R., Agüero, C., "Lógica, ciencia y experiencia en la valoración de la prueba", en Revista Chilena de Derecho, vol. 41, No 2, 2014.

${ }^{70}$ Un ejemplo de ello son los conceptos fundamentales de Kelsen (el concepto de sanción determina lo que es el concepto de delito, este último, el de responsabilidad y así, sucesivamente). 
Duce, M., "Algunas lecciones a partir de cuatro casos de condena de inocentes en Chile", en Revista de Derecho de la Universidad Católica del Norte, Año 22, Nº 1, 2015.

FuenZalida, E., "Investigación sociojurídica, cultura política y generaciones en Chile: un estudio exploratorio", en Anuario de Filosofía Jurídica y Social, 1998, N 16, pp. 337-368.

Gascón, M., Los hechos en el Derecho. Bases argumentales de la prueba. Marcial Pons, Madrid, 1999.

Goldman, A., "A guide to Social Epistemology”, en Goldman, A., Social Epistemology. Essential Readings, Oxford University Press, Oxford, 2011.

Grafton, A., Los orígenes trágicos de la erudición, Fondo de Cultura Económica, México, 2015.

Gross, J., The Oxford Book of Aphorisms, Oxford University Press, Oxford, (reimpresión), 2010.

HaAck, S., Evidence Matters: Science, Proof, and Truth in the Law, Cambridge University Press, New York, 2014.

KuHN, Th., "La tensión esencial: tradición e innovación en la investigación científica", en Kuhn, Th., La tensión esencial. Estudios selectos sobre la tradición y el cambio en el ámbito de la ciencia, FCE, Madrid, 1993 (2 ${ }^{\mathrm{a}}$ reimp.).

Laudan, L., Verdad, error y proceso penal. Un ensayo sobre epistemología jurídica, Marcial Pons, Madrid, 2013.

Lempert, R., "The New Evidence Scholarship: Analyzing the Process of Proof”, en 66 Boston University Law Review, 1986.

PARK, R., Saks, M., "Evidence Scholarship Reconsidered: Results of the Interdisciplinary Turn”, en 47 Boston College Law Review, 2006.

Stein, A., "Contra la prueba libre", en Revista de Derecho (Valdivia), vol. XXVI, N², 2013.

Twining, W., "Moving Beyond Law: Interdisciplinarity and the Study of Evidence", en Dawid, P., Twining, W., Vasilaki, M. (eds.), Evidence Inference and Enquiry, The Bristish AcademyOxford University Press, 2011.

Twining, W., "Taking Facts Seriously-Again”, en Twining, W., Retinking Evidence, Cambridge University Press, Cambridge, $2^{\mathrm{a}}$ edición, 2006.

TwINING, W., Theories of Evidence: Bentham \& Wigmore, Weidenfeld \& Nicolson, London, 1985.

WALLERSTEIN, I., "La antropología, la sociología y otras disciplinas dudosas", en Wallerstein, I., Las incertidumbres del saber, Gedisa, Barcelona, 2005. 